\title{
SOBRE LOS MODIFICADORES DE APROXIMACIÓN Y PRECISIÓN*
}

\author{
RaQuel GonzÁlez Rodríguez \\ (Universidad de Castilla-La Mancha) \\ Raquel.Gonzalez@uclm.es
}

\begin{abstract}
Resumen
En este trabajo proponemos que los modificadores de aproximación son términos de polaridad positiva, es decir, que son incompatibles con la negación, mientras que los de precisión no lo son. Se describen los contextos en los que los modificadores de aproximación son rechazados $\mathrm{y}$, a partir del análisis semántico de los modificadores de aproximación y precisión desarrollado por U. Sauerland y P. Stateva (en prensa), se explica por qué los primeros son incompatibles con la negación y los segundos compatibles con ella.

PALABRAS CLAVE: vaguedad escalar, modificadores de aproximación y precisión, negación, términos de polaridad positiva.
\end{abstract}

\begin{abstract}
In this paper I propose that modifiers of approximation are positive polarity items, that is, that they cannot occur with negation, while modifiers of precision are not positive polarity items. I describe the contexts in which modifiers of approximation are rejected and, based on the semantic analysis of modifiers of approximation and precision developed by U. Sauerland y P. Stateva (in press), I offer a proposal which explains why the former are positive polarity items and while the latter are not incompatible with negation.

KEYWORDS: scalar vagueness, modifiers of approximation and precision, negation, positive polarity items.
\end{abstract}

\section{Introducción}

Los modificadores de aproximación y precisión se caracterizan por que "las entidades sobre las que inciden pierden su capacidad denotadora para adquirir la que obtienen del lugar que se les asigna en ciertas escalas" (Bosque, 1989: 144). Se trata de elementos acotadores que no modifican la intensión del sintagma al que modifican, sino su extensión (Lakoff, 1972; Moreno Cabrera, 1984; Bosque, 1989)'. En (1a) se recoge una muestra representativa de los modificadores de aproximación; en (1b) se ejemplifica el paradigma de los modificadores de precisión:

(1) a. alrededor de tres horas, casi cien reos, aproximadamente cinco kilos, cuatro metros escasos, unos ocho hombres, más o menos veinte diputados

b. exactamente un milímetro, media hora exacta, dos toneladas justas

\footnotetext{
* Agradezco a I. Bosque, Ángeles Carrasco, Vicky Escandell, María Jesús Fernández Leborans, Luis García e Isabel Pérez sus comentarios sobre el contenido y/o los datos de este trabajo. Cualquier error es, por supuesto, únicamente a mí atribuible.

1 Sobre los cuantificadores de aproximación y precisión en español, véanse J. García-Medall (1993) y M. García-Page (1995), donde se exponen algunas de sus propiedades sintácticas y semánticas. En lo que respecta a la semántica de estos elementos, consúltense P. Lasersohn (1999) y U. Sauerland y P. Stateva (en prensa).
} 
Como señala I. Bosque (1989: 144), existe una diferencia entre los adverbios y los adjetivos que integran esta clase de modificadores: mientras que los segundos únicamente pueden incidir sobre sintagmas cuantificados (cfr. *ciego escaso, *eso exacto, etc.), los primeros son capaces de acompañar a otra clase de sintagmas (cfr. casi ciego, exactamente eso, etc.). Nosotros nos centraremos en aquellos casos en que los modificadores de aproximación y precisión inciden sobre sintagmas cuantificados haciendo referencia a un valor escalar cercano o idéntico, respectivamente, al denotado por el constituyente al que acompañan, como ilustran todos los ejemplos de (1). Dentro de los que apuntan a un valor cercano al introducido por el elemento al que modifican, es decir, de los de aproximación, es posible distinguir tres clases dependiendo del intervalo de la escala al que se asocien (J. García-Medall, 1993): (a) aproximativos neutros: aquellos que pueden referir tanto a un valor superior como a uno inferior al denotado por el elemento modificado (cfr. alrededor de veinte canciones, aproximadamente siete cazadores, unos mil afectados, más o menos quince juicios, etc.); (b) aproximativos defectivos: los que señalan a un valor inferior (cfr. casi tres dias, prácticamente cien concejales, cuatro metros escasos, etc.); (c) aproximativos excesivos: aquellos que aluden a un valor superior (cfr. un kilómetro largo, tres horas y pico, etc.) ${ }^{2}$.

En este trabajo estudiaremos una propiedad de los modificadores de aproximación y precisión que, en lo que se nos alcanza, no había sido observada con anterioridad y que, como demostraremos, está estrechamente relacionada con sus propiedades semánticas: su (in)compatibilidad con la negación. Como muestra el contraste entre (2) y (3), los modificadores de aproximación (cfr. (2)) no pueden estar en el alcance de la negación, de forma que constituyen términos de polaridad positiva, mientras que los modificadores de precisión no presentan esa restricción (cfr. (3)) $)^{3}$ :

(2) a. (*No) llegaron aproximadamente a las cuatro.

b. (*No) recorrió casi cuatro metros.

c. (*No) le esperaron apenas diez minutos.

(3) a. No llegaron exactamente a las cuatro.

b. No recorrió cuatro metros justos.

Las oraciones de (2) resultan gramaticales si se interpretan como enunciados eco, es decir, como respuesta a la correspondiente oración afirmativa, pero no en otro caso, como muestra el hecho de que no puedan comenzar una conversación o un relato (Bosque, 1980b: 20). La negación se interpreta como externa y refuta una proposición previa al expresar su desacuerdo con la realidad (Horn, 1985); por ejemplo, una oración como (2a) se parafrasearía como 'No es cierto que llegaran aproximadamente a las cuatro'. Al tratarse de enunciados eco que niegan una proposición anterior, las oraciones con negación externa pueden contener términos de polaridad positiva. Pues bien, esta interpretación debe ser descartada a lo largo de todo este trabajo, a menos que se señale explícitamente lo contrario.

2 J. García-Medall (1993) distingue, además, varias subclases dentro de los aproximativos que integran cada uno de los dos últimos grupos a partir del tipo de inferencias que realizan.

3 Nótese que en las oraciones de (3) estamos ante una negación de constituyentes, puesto que lo refutado es el valor introducido por el modificador de precisión, es decir, que la expresión numeral deba interpretarse de forma precisa. 
Aunque las tres clases de modificadores aproximativos señaladas son incompatibles con la negación (cfr. (2)), dejaremos de lado el estudio de los aproximativos defectivos (cfr. (2b)) y de exceso (cfr. (2c)). La razón es que los modificadores pertenecientes a estas clases presentan un comportamiento más complejo que los aproximativos neutros (cfr. (2a)), puesto que no siempre rechazan la presencia de la negación. Como hemos visto, todos ellos pueden modificar a expresiones numerales, casos en los que no pueden concurrir con la negación (cfr. (2)). Sin embargo, la situación varía si observamos qué sucede cuando inciden sobre un nombre, las palabras negativas nadie, ningín y nada o un sintagma verbal. Obsérvense los datos de (4) y (5):

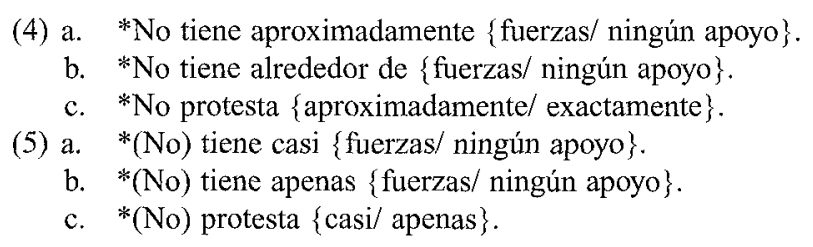

Los aproximativos neutros no pueden acompañar a esa clase de elementos, independientemente de si está presente o no una negación (cfr. (4)). Los aproximativos defectivos y los de exceso, en cambio, pueden modificar a los constituyentes señalados y, además, en esos casos no rechazan la presencia de la negación, sino que la exigen, pasando a ser términos de polaridad negativa (cfr. (5) $)^{4}$. El estudio de estos últimos modificadores requiere un análisis que abarque no solo su comportamiento como términos de polaridad positiva (cfr. (2b) y (2c)), sino también el que presentan como términos de polaridad negativa (cfr. (5)), estableciendo la relación que existe entre ambos usos. Puesto que, como hemos señalado, nuestro propósito es explicar por qué los modificadores de aproximación, a diferencia de los modificadores de precisión, son términos de polaridad positiva, es decir, por qué resultan incompatibles con la negación, nos centraremos en aquellos que presentan un comportamiento uniforme a este respecto: los aproximativos neutros ${ }^{5}$.

El trabajo está dividido como sigue. En primer lugar, presentaremos el análisis semántico de los modificadores de aproximación y precisión desarrollado por U. Sauerland y P. Stateva (en prensa). En segundo lugar, abordaremos el comportamiento que presentan dichos modificadores con respecto a los contextos negativos. Para ello, describiremos los entornos en los que los modificadores de aproximación son rechazados y, a partir del análisis semántico de los modificadores de aproximación y precisión que proponen U. Sauerland y P. Stateva (en prensa), explicaremos por qué los primeros son incompatibles con la negación, mientras que los segundos no. En tercer lugar, expondremos las conclusiones del trabajo.

\footnotetext{
4 Cuando el modificador acompaña a una palabra negativa, es esta la que exige la presencia de la negación. Pero a pesar de que en estos contextos no podemos sostener que el modificador se comporte como un término de polaridad negativa, sigue sin rechazar la presencia de la negación, a diferencia de lo que ocurre en (3).

5 Para mayor simplicidad, con el término modificadores de aproximación aludiremos a partir de ahora exclusivamente a los neutros.
} 


\section{La semántica de los modificadores de aproximación y precisión: U. Sauerland y $P$. Stateva (en prensa)}

U. Sauerland y P. Stateva (en prensa) proponen un análisis semántico de los modificadores de aproximación y precisión basado en las distintas lecturas a las que puede dar lugar una expresión numeral. Estos lingüistas parten del trabajo de M. Krifka (2007), en el que se estudia la ambigüedad de las expresiones numerales, que pueden interpretarse de forma más o menos precisa. Así, si bien es cierto que en una oración como, por ejemplo, la de (6), el constituyente doscientas personas puede interpretarse como 'exactamente doscientas personas', la expresión numeral tiende a recibir una lectura más relajada en la que equivale a 'aproximadamente doscientas personas':

(6) En el concierto había doscientas personas.

La lectura más prominente es la de 'aproximadamente doscientas personas' debido a que, si tenemos en cuenta un sistema decimal, el numeral empleado constituye un valor destacado en la escala de medición. A partir de ese valor es posible referir a otros menos destacados y que son cercanos a él, como, por ejemplo, los contenidos en el intervalo que va de ciento noventa a doscientos diez. Si, por el contrario, se emplea una expresión numeral que no constituye un valor destacado dentro del sistema de medición usado, la interpretación que obtenemos es exclusivamente aquella en la que el numeral se interpreta de forma precisa. En (7), por ejemplo, la lectura que surge por defecto es la correspondiente a una paráfrasis como 'En el concierto había exactamente ciento noventa y ocho personas':

(7) En el concierto había ciento noventa y ocho personas.

Según M. Krifka (2007), la imposibilidad de interpretar ciento noventa y ocho, frente a doscientos, de forma aproximada responde a principios pragmáticos. Si un hablante profiriera una oración como la de (7) teniendo en consideración una lectura aproximada del numeral, haría alusión a un valor comprendido, por ejemplo, entre ciento noventa y cinco y doscientos uno. Sin embargo, dicho valor puede ser referido igualmente por la interpretación aproximada de una expresión formalmente más simple, doscientos, lo que impide el uso aproximado de la más compleja, ciento noventa y ocho. El que la interpretación aproximada de un numeral sea posible únicamente en aquellos casos en que constituye una forma destacada dentro de la escala empleada responde, en consecuencia, a una preferencia más general por el uso de las formas simples frente a las complejas cuando la información que se obtiene es equivalente ${ }^{6}$. La lectura precisa, por su parte, está disponible en todos los numerales porque no hay otros que expresen lo mismo si nos atenemos a dicha interpretación.

De acuerdo con la lectura imprecisa, la expresión numeral no señala, como hemos dicho, el valor que le corresponde en la escala, sino un intervalo de la escala. La longitud de ese intervalo, es decir, el número de valores que lo integran, dependerá de cómo esté segmenta-

6 Como señala M. Krifka (2007), una expresión es (o no) simple dependiendo de la escala a la que se asocie. Así, mientras que veinticuatro resulta una forma compleja si nos atenemos a una escala en la que se cuantifica el número de individuos que asisten a un concierto, constituye una forma simple en caso de que se estén computando horas, ya que esa es la cantidad de horas que tiene un día. 
da la escala; en otras palabras, dependiendo de la precisión que se asigne a una determinada escala, esta será dividida de forma distinta, puesto que los valores que se toman en consideración varían. Al calcular las personas que asisten a un concierto, uno podría basarse en una escala centesimal, donde los valores relevantes son los correspondientes a las centenas (cfr. (8a)), una escala más precisa en donde los valores tomados en consideración son, por ejemplo, los señalados en ( 8 b), etc.:

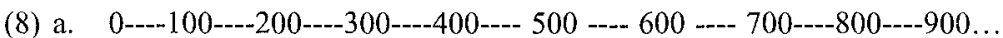

$$
\begin{aligned}
& \text { b. } 0 \text {-- } 50 \text {-- } 100 \text { - } 150 \text {-- } 200 \text {-- } 250 \text { - } 300 \text {-- } 350 \text {-- } 400 \text {-- } 450 \text {-- } 500 \text {-- } 550 \ldots
\end{aligned}
$$

Nótese que si el cálculo se realiza sobre una escala como la de (8a), el numeral doscientos, además de referir de forma precisa al valor escalar que le corresponde, puede obtener una lectura imprecisa según la cual señalaría el intervalo que va de ciento cincuenta a doscientos cincuenta y, por tanto, a cualquiera de los valores que forman parte de él. En (8b), en cambio, ese mismo numeral señalará, en una lectura imprecisa, un intervalo menor, el que se extiende desde ciento setenta y cinco hasta doscientos veinticinco. La razón es que la longitud que existe entre los valores tomados en consideración para llevar a cabo el cálculo difiere de una escala a otra: mientras que en la primera se consideran relevantes los valores correspondientes a las centésimas, en la segunda se tienen en cuenta valores inferiores. A esta propiedad de las escalas, es decir, a la longitud que existe entre los valores relevantes, se la denomina granularidad (Krifka, 2007). La granularidad de una escala hace referencia, por tanto, a la densidad de la escala, es decir, a la forma en que está segmentada.

Tomando como punto de partida el trabajo de M. Krifka (2007), U. Sauerland y P. Stateva (en prensa) consideran que la granularidad es un parámetro contextual de interpretación, puesto que fija el intervalo de la escala al que puede referir una expresión numeral. Una función de granularidad relaciona un punto o valor de una escala con un intervalo que lo contiene. Dado que las escalas pueden ser segmentadas de forma distinta, al interpretar una expresión numeral varias granularidades deben ser tenidas en consideración. De este modo, dependiendo de la granularidad con respecto a la que se valore la escala, un numeral como, por ejemplo, siete, podrá hacer referencia a los siguientes intervalos ${ }^{7}$ :

$$
\begin{aligned}
\text { (9) a. } & \operatorname{gran}_{\text {sutil }}(7):\{7\}^{8} \\
\text { b. } & \operatorname{gran}_{\text {medio }}(7):\{6,90,6,91,6,92, \ldots ., 7, \ldots, 7,08,7,09,7,10\} \\
\text { c. } & \operatorname{gran}_{\text {basto }}(7):\{5,50,5,51,5,52, \ldots \ldots, 7, \ldots ., 8,48,8,49,8,50\}
\end{aligned}
$$

Las lecturas de (9a), (9b) y (9c) surgen si la granularidad es sutil, media y basta, respectivamente. Lo que establece el parámetro de granularidad $\mathrm{y}$, por tanto, distingue a esas lecturas, es el intervalo de la escala con respecto al que se interpreta el numeral. Cuanto más sutil sea la granularidad, más restringido será el intervalo de la escala al que refiere el nume-

7 A lo largo de este trabajo emplearemos los siguientes símbolos y abreviaturas: [[ ]] la denotación de la expresión que aparezca en su interior; = símbolo de igualdad; 1 verdadero; $\vee$ disyunción (léase 'o...'); $\neg$ negación; \{\} definición de un conjunto; gran 'granularidad'; P 'proposición'; ssi 'si y solo si'.

8 En realidad, U. Sauerland y P. Stateva (en prensa) consideran que esta granularidad engloba no solo a la cantidad denotada por el numeral sino también a otras cercanas a él: $\operatorname{gran}_{\text {sutil }}(7):\{6,99, \ldots \ldots, 7, \ldots ., 7,01\}$. Aquí asumiremos, sin embargo, que la granularidad sutil es la que restringe totalmente la interpretación del numeral, ya que esa es la lectura más precisa posible. 
ral. De este modo, cuando un hablante expresa una oración como La mesa pesa siete kilos, es posible interpretar que el peso de la silla es alguno de los contenidos en el intervalo de $(9 \mathrm{a}),(9 \mathrm{~b})$ o $(9 \mathrm{c})$, dependiendo del parámetro de granularidad que se tenga en consideración. La denotación del numeral es más restringida cuando la granularidad es sutil que cuando es media o basta y, del mismo modo, la granularidad media restringe la interpretación del numeral más que la basta.

La vaguedad escalar que presentan las expresiones numerales está, según U. Sauerland y P. Stateva (en prensa), estrechamente vinculada con la función que realizan los modificadores de aproximación y precisión ${ }^{9}$. Estos modificadores contribuyen a la denotación de la expresión numeral a la que modifican fijando el parámetro de granularidad con respecto al que se evalúa. Los modificadores de precisión, que restringen la denotación de la expresión numeral, esto es, refieren a un valor idéntico al denotado por el elemento modificado, fuerzan a interpretar el numeral con respecto al parámetro de granularidad más sutil (cfr. (10a)). Los modificadores de aproximación, que aluden a un valor cercano al introducido por el numeral al que modifican, haciendo su denotación más imprecisa, obligan a interpretar el numeral en relación con el parámetro de granularidad más basto (cfr. (10b)):

(10) a. $[[\text { exactamente }]]^{\text {gran }}(G)=G(\{$ el más sutil(gran $\left.)\}\right)$

b. [[aproximadamente] ] $]^{\text {gran }}(\mathrm{G})=\mathrm{G}(\{$ el más basto $($ gran $)\})$

(Sauerland y Stateva, en prensa)

El análisis de los modificadores de aproximación y precisión recogido en (10) permite dar cuenta de la diferencia existente entre las oraciones de (11):

(11) a. Han recogido mil fresas.

b. Han recogido exactamente mil fresas.

c. Han recogido aproximadamente mil fresas.

La construcción de (11a) admite, como ya hemos indicado, tanto una lectura precisa como una imprecisa del numeral, dependiendo de cuál sea la granularidad relevante. De acuerdo con la interpretación precisa, el número de fresas recogidas ha de ser mil, sin que exista posibilidad de que se haya recogido ni una más ni una menos. En la lectura imprecisa, la oración simplemente expresa que la cantidad de fresas recogidas ronda las mil, pudiendo ser unas cuantas más o menos de las denotadas por ese numeral. En (11b) y (11c), sin embargo, la ambigüedad del numeral desaparece como consecuencia de la modificación realizada por el adverbio. En la primera de esas construcciones, exactamente, siguiendo a U. Sauerland y P. Stateva (en prensa), provoca que el numeral se interprete de acuerdo con el parámetro de granularidad nás sutil (cfr. (10a)). De ello se sigue que, en (11b), la única posibilidad sea interpretar el numeral de manera precisa. En la segunda de ellas, aproxima-

9 Estos autores distinguen entre vaguedad escalar y vaguedad epistémica. Los modificadores que estamos estudiando interactúan con la vaguedad escalar, mientras que modificadores como seguramente, probablemente, etc. lo hacen con la epistémica. Otros autores, sin embargo, consideran que la vaguedad escalar es un caso de imprecisión, mientras que la epistémica sí se adscribe al fenómeno de la vaguedad. Sobre las nociones de 'vaguedad' e 'imprecisión', consúltense, entre otros, T. Williamson (1994), M. Pinkal (1995), P. Lasersohn (1999) y C. Kennedy (2007). 
damente fuerza que el parámetro de granularidad relevante sea el más basto, lo que explica que solo obtengamos la lectura imprecisa del numeral. En (12) ilustramos las posibilidades interpretativas del numeral de (11) mediante las distintas granularidades:

(12) a. Han recogido mil fresas.

$[[\mathrm{mil}]]^{\text {gran sutil }}=\{1000\}$

$[[\text { mil }]]^{\text {gran medio }}=\{995,996, \ldots, 1000, \ldots, 1004,1005\}$

$[[\text { mil }]]^{\text {gran basto }}=\{990,991, \ldots, 1000, \ldots, 1009,1010\}$

b. Han recogido exactamente mil fresas.

$[[\mathrm{mil}]]^{\text {el más sutil(gran })}=\{1000\}$

c. Han recogido aproximadamente mil fresas.

$[[\mathrm{mil}]]^{\text {el más basto(gran) }}=\{990,991, \ldots, 1000, \ldots, 1009,1010\}$

Según U. Sauerland y P. Stateva (en prensa), la función de los modificadores de aproximación y precisión es establecer la granularidad con respecto a la cual debe interpretarse el numeral sobre el que inciden. Los primeros, entre los que están aproximadamente, alrededor de, etc., que hacen más imprecisa la denotación del numeral, establecen como parámetro de granularidad relevante el más basto; los segundos, que restringen el significado del numeral, como, por ejemplo, exactamente, fijan como granularidad la más sutil ${ }^{10}$.

\section{La distribución de los modificadores de aproximación y precisión en contextos negativos}

En este apartado ofreceremos una explicación de las propiedades distribucionales de los modificadores de aproximación y precisión y, más en concreto, de que los primeros sean rechazados en ciertos contextos negativos mientras que los segundos no. Nuestro primer cometido será determinar en qué entornos son rechazados los modificadores de aproximación. Una vez descritos esos contextos, desarrollaremos un análisis que dé cuenta tanto de que los modificadores de aproximación sean términos de polaridad positiva como de que los de precisión no lo sean.

\subsection{La distribución de los modificadores de aproximación en contextos negativos}

Los términos de polaridad positiva son aquellas unidades léxicas o sintagmas que no pueden aparecer en el alcance de la negación; las expresiones en cursiva de (13), por ejemplo, son términos de polaridad positiva, puesto que su concurrencia con la negación desencadena la mala formación de la oración (descártese la lectura de negación externa):

(13) a. *No hemos llegado ya.

b. * La película no me ha gustado bastante.

c. *No son prácticamente las tres.

10 Otra prueba a favor de esta propuesta es que en aquellos casos en que el numeral, al ser una expresión compleja, únicamente admite una lectura precisa, resulta anómala la modificación mediante aproximadamente, pero no la de exactamente, auque la presencia de este modificador sea redundante:

(i) a. \#Han recogido aproximadamente noventa y ocho fresas.

b. Han recogido exactamente noventa y ocho fresas. 
d. *No conozco a Juan desde que teníamos cinco años.

e. *No hace un frio de mil diablos.

f. *No tiene Vd. toda la razón del mundo.

(Bosque, 1980b: 20)

Los modificadores de aproximación, al igual que las expresiones de (13), son incompatibles con la negación y, por tanto, constituyen términos de polaridad positiva, como ilustran los ejemplos de $(14)^{11}$ :

(14) a. *No han visitado aproximadamente cincuenta oficinas.

b. *No levantó unos cuarenta kilos.

Aunque la agramaticalidad de las construcciones de (14) muestra que los modificadores de aproximación resultan incompatibles con la negación, cabe señalar que estos pueden concurrir con ella si se cumple cierta condición. Obsérvese a este respecto el contraste de gramaticalidad que presentan las oraciones de (15) y (16), a pesar de que en ambos casos el modificador concurre con una negación:

(15) a. *No ha ido aproximadamente a ciento cincuenta kilómetros por hora.

b. *Alberto no se gastó aproximadamente cien euros.

(16) a. No ha ido aproximadamente a ciento cincuenta kilómetros por hora para que le pusieran una multa.

b. Alberto no se gastó aproximadamente cien euros porque tuviera que saldar una deuda.

La única diferencia que presentan las construcciones de (15) y (16) es la presencia de una cláusula final o una causal en subjuntivo en las segundas. Las cláusulas subordinadas en subjuntivo se caracterizan, entre otras cosas, por que cuando aparecen dentro del ámbito sintáctico de la negación deben ser obligatoriamente el elemento refutado (Bosque, 1990; Laka, 1990; Quer, 1998; Borgonovo, 2002; entre otros). Ello queda probado por la imposibilidad de introducir un sintagma correctivo que fuerce a otro constituyente a ser el foco de la negación, como se muestra en (17):

(17) a. Sus amigas no han ido al cine [Foco para que Juan se quede solo], *sino al teatro. b. Malena no ha comprado un ordenador $\left[_{\mathrm{F} o \mathrm{co}}\right.$ porque tenga el suyo estropeado], *sino un televisor.

Lo que diferencia a las oraciones de (15) y (16) es, por tanto, que, en las primeras, los modificadores de aproximación, además de estar en el ámbito de la negación, son su foco, es decir, los elementos refutados, mientras que, en las segundas, el foco de la negación es la cláusula subordinada. El que el elemento refutado no es el modificador de aproximación cuando está presente una cláusula final o una causal en subjuntivo lo muestran los ejemplos de (18), donde se ha añadido un sintagma correctivo que determina cuál es el foco de la negación:

11 De hecho, nótese que uno de los ejemplos de I. Bosque (1980b: 20) ilustra que el adverbio prácticamente, que es un modificador aproximativo de defecto, se comporta como un término de polaridad positiva (cfr. (13c)). 
(18) a. No ha ido aproximadamente a ciento cincuenta kilómetros por hora para que le pusieran una multa, sino para llegar antes.

b. Alberto no se gastó aproximadamente cien euros porque tuviera que saldar una deuda, sino porque le habían subido el sueldo.

De estos datos se desprende que los modificadores de aproximación son rechazados en una oración negativa cuando constituyen el foco de la negación (cfr. (15)), pero no si, a pesar de estar en el ámbito de la negación, otro constituyente es el refutado (cfr. (16)). En este sentido, es necesario observar qué sucede cuando la refutación de un determinado constituyente se expresa a través de la preposición sin, y no de la partícula $n o^{12}$. De acuerdo con nuestra descripción, los modificadores de aproximación deberían poder aparecer en el término de sin siempre y cuando no sean el elemento refutado por dicha preposición. Para comprobar si la caracterización que hemos propuesto es adecuada, analizaremos el comportamiento de los modificadores de aproximación con respecto a la preposición sin en dos situaciones distintas: cuando la preposición selecciona una cláusula que contiene un modificador de aproximación y cuando selecciona directamente al sintagma encabezado por un modificador de esa clase. Como se muestra en (19), si la preposición sin selecciona una oración, esta puede contener un modificador de aproximación sin que ello desencadene la mala formación de la construcción:

(19) María se arregló sin tardar aproximadamente dos horas.

La gramaticalidad de esta construcción constituye a primera vista un contraejemplo a la descripción de la distribución de los modificadores de aproximación que hemos establecido. Si son rechazados cuando constituyen el foco de la negación, la oración de (19) debería ser agramatical. El constituyente introducido por sin parece semánticamente equivalente a 'No tardó aproximadamente dos horas', donde el foco de la negación es el adverbio. Pero a pesar de que aparentemente no hay ningún constituyente distinto del modificador de aproximación que sea el foco de la negación, consideramos que lo refutado por la preposición $\sin$ no es el modificador. A este respecto, nos gustaría sugerir que en las cláusulas de infinitivo encabezadas por $\sin$ lo que se niega es la relación semántica que mantiene la cláusula matriz con la introducida por la preposición. Esta hipótesis se basa en el trabajo de I. Bosque (1980a: 82 ), donde se propone, entre otras cosas, que las oraciones de (20) no son sinónimas:

(20) a. Lo perderás de vista no prestándole atención. b. Lo perderás de vista sin prestarle atención.

(Bosque 1980a: 82)

I. Bosque (1980a) observa que ambas construcciones poseen un sentido modal, según el cual admiten una paráfrasis como "No prestarle atención es el modo de perderlo de vista"13.

12 También contienen una negación las palabras negativas en posición preverbal, puesto que estas confieren valor negativo a toda la oración (Bosque, 1980b). Sin embargo, no se refuta ningún otro constituyente oracional, sino que ellas mismas son el foco de la negación; en otras palabras, contienen una negación que recae sobre ellas (cfr. Ninguno de esos actores ha cenado en mi restaurante, *sino en el tuyo).

13 Como I. Bosque (1980a: 82) señala, la oración de (20a) puede interpretarse también como una negación de constituyentes. La paráfrasis correspondiente a esa lectura sería 'Lo perderás de vista no prestándole atención, sino marchándote'. 
Lo que diferencia a esas oraciones es que, en (20b), el ámbito de la negación abarca a un predicado de necesidad, pero, en (20a), ese predicado queda fuera del ámbito de la negación. En otras palabras, en (20a) se expresa que para perderlo de vista es necesario no prestarle atención; (20b), por su parte, denota que para perderlo de vista no es necesario prestarle atención. De acuerdo con esta propuesta, la negación incide directamente sobre la cláusula en las estructuras del tipo ' $n o+$ gerundio', mientras que, en las secuencias del tipo 'sin + infinitivo', la negación afecta a la relación modal que se establece entre la cláusula matriz y la subordinada ${ }^{14}$.

Pues bien, si tenemos en cuenta esta propuesta, el que los modificadores de aproximación no sean rechazados cuando aparecen en el término de la preposición $\sin$ (cfr. (19)) no constituye un contraejemplo a nuestra descripción. La razón es que el foco de la negación no es, según ese análisis, el modificador, sino la relación de implicación que se da entre la cláusula matriz y la subordinada. Así, en (19) no se niega que el tiempo que María tarda en arreglarse sea aproximadamente dos horas, sino que el contenido proposicional de la cláusula matriz implique necesariamente el de la seleccionada por $\sin$. Al no ser el elemento refutado, el modificador de aproximación no resulta incompatible con la negación introducida por la preposición, tal como predice la descripción que hemos realizado de la limitada distribución de dichos elementos.

En lo que respecta a aquellos casos en que sin selecciona directamente un constituyente encabezado por un modificador de aproximación, la secuencia resultante es agramatical ${ }^{15}$ :

(21) a. *sin alrededor de \{veinte estudiantes/ dos kilos de manzanas....

b. *sin aproximadamente \{veinte estudiantes/ dos kilos de manzanas...

c. *sin unos \{veinte estudiantes/ dos kilos de manzanas...

Al no haber ningún otro constituyente que forme parte del término de la preposición, la única posibilidad es que el modificador de aproximación sea el foco de sin, pero, como se trata de un término de polaridad positiva, la construcción resulta agramatical. Nótese, no obstante, que los sintagmas de (21) son aceptables si constituyen construcciones absolutas adjuntas a la oración:

(22) a. Sin alrededor de diez alumnos, no se va a aprobar el grado.

b. Sin alrededor de mil firmas en contra, la propuesta seguirá adelante.

14 Sobre los casos en que 'sin + infinitivo' podría sustituirse por y no (cfr. (i)), véase I. Bosque (1980a):

(i) a. Se marchó y no se despidió de ella.

b. Se marchó sin despedirse de ella. (Bosque, 1980a: 83, 84)

15 Cuando casi y apenas modifican a un nombre (cfr. (ia)), a las palabras negativas ningún, nada y nadie (cfr: (ib)) o a un sintagma verbal (cfr. (ic)), sí que pueden ser término de la preposición sin:

(i) a. $\sin \{$ casi/ apenas $\}$ fuerzas

b. $\sin \{$ casi/ apenas $\}$ ningún apoyo

c. $\sin \{$ casi/ apenas $\}$ protestar

Sin embargo, estas construcciones no constituyen un contraejemplo a la descripción realizada. Como señalamos en la introducción, la distribución de estos aproximativos cuando modifican a un elemento que no es una expresión numeral difiere de aquella en que inciden sobre esa clase de constituyentes. 
Pero, de nuevo, la gramaticalidad de estas construcciones no invalida la propuesta de que los modificadores de aproximación no pueden ser el foco de la negación. De forma paralela a lo que sucede cuando $\sin$ selecciona una cláusula que contiene un modificador de este tipo, el foco de la preposición en (22) no es el modificador; dicho de otro modo, el sintagma introducido por alrededor de no es el constituyente refutado por la negación. Cuando se emplea en una construcción absoluta, sin, al igual que con, tiene valor de predicado existencial, que es el refutado por la negación; en otras palabras, los constituyentes introducidos por $\sin$ en (22) equivalen a \{No habiendo/ si no hay\} alrededor de diez alumnos matriculados, no se va a aprobar el grado y \{No habiendo/ si no hay\} alrededor de mil firmas en contra, la propuesta seguirá adelante, donde el foco de la negación es el predicado existencial, y no el modificador de aproximación.

Antes de acabar esta sección, quisiéramos observar también cuál es el comportamiento de los modificadores de aproximación en los entornos negativos creados por elementos distintos de no y sin. Existen otras construcciones que crean contextos negativos, puesto que, al igual que la negación, legitiman la presencia de un término de polaridad negativa en la oración (Bosque, 1980b). Nos estamos refiriendo a los cuantificadores y adverbios cuantitativos poco(s), solo, raramente, apenas, las palabras negativas (cfr. ningún, nadie, etc.), las locuciones prepositivas antes de, en lugar de y en vez de, ciertos predicados de significado negativo (cfr. dudar, negar, etc.) y factivos emotivos (cfr. sorprenderse, molestar, etc.), las oraciones interrogativas y exclamativas retóricas, las construcciones comparativas y las superlativas y los ordinales primero y último. Los siguientes ejemplos, tomados de I. Bosque (1980b), ponen de manifiesto la posibilidad que poseen todos estos elementos de actuar como inductores de polaridad negativa, ya que legitiman los términos de polaridad negativa que aparecen en cursiva:

(23) Cuantificadores y adverbios cuantitativos

a. Poca gente entendería nada de lo que dices.

b. Sólo Juan ha conseguido pegar ojo.

c. Raramente dice nada.

d. Apenas vino nadie.

(24) Palabras negativas

Nadie quiere nada.

(25) Locuciones prepositivas

a. Antes de (hacer) nada, debes lavarle las manos.

b. En lugar de intentar nada ahora, es mejor esperar a más tarde.

(26) Predicados negativos y factivos emotivos

a. Dudo que se haya enterado nadie.

b. Me indigna que venga nadie.

(27) Oraciones interrogativas y exclamativas retóricas.

a. ¿Cuándo tme ha regalado nada?

b. ¿Qué excursión al campo ni qué narices!

(28) Construcciones comparativas, superlativas y los ordinales primero y último

a. Juan ha llegado más tarde que nunca.

b. Es la última vez que te digo nada.

c. Fue la primera persona a la que vi mover un dedo por Juan. 
Pues bien, a pesar de que estos elementos desencadenan contextos negativos, los modificadores de aproximación no presentan ninguna restricción en lo que respecta a su posibilidad de concurrir con ellos, como ilustra la buena formación de las siguientes oraciones:

(29) Cuantificadores y adverbios cuantitativos ${ }^{16}$

a. Pocos recién nacidos pesan alrededor de cinco kilos.

b. Solo María mide alrededor de 1'70 metros.

c. Su madre raramente compra alrededor de cinco kilos de patatas.

(30) Palabras negativas

Ninguno de esos muebles mide alrededor de tres metros.

(31) Locuciones prepositivas

a. Antes de hacer alrededor de mil copias, debería haberse enterado de cuántas eran necesarias.

b. En lugar de esperar alrededor de dos horas a que llegaras, tendría que haber empezado a trabajar.

(32) Predicados negativos y factivos emotivos

a. Dudo que pese alrededor de sesenta kilos.

b. Me indigna que haya desperdiciado alrededor de cien hojas.

(33) Oraciones interrogativas y exclamativas retóricas.

a. ¿Cuándo has ganado tú alrededor de cuatro mil euros?

b. ¿Qué va a medir ese alrededor de dos metros!

(34) Construcciones comparativas, superlativas y los ordinales primero y último

a. Ese violinista tiene más conciertos programados que alrededor de quince de sus compañeros de orquesta.

b. Es la secretaria más competente que ha tenido en alrededor de diez años.

c. Es la primera persona que le hace correr alrededor de doce kilómetros sin parar.

Los datos expuestos en este apartado indican que los modificadores de aproximación son sensibles exclusivamente al foco de la negación, de forma que pueden concurrir con la partícula no y la preposición sin siempre y cuando no sean el elemento refutado por ellas. Además, estos modificadores no muestran ningún tipo de incompatibilidad con el resto de construcciones que crean entornos negativos.

\subsection{La distribución de los modificadores de aproximación y precisión en contextos negativos}

En el apartado anterior hemos mostrado que los modificadores de aproximación no pueden ser el foco de la negación; los modificadores de precisión, en cambio, no resultan incompatibles con la negación, como indicamos en la introducción. Nuestra propuesta a este respecto consiste en deferider que la distribución de esos elementos obedece a razones semánticas y, en concreto, a que sus propiedades léxicas son (in)compatibles con las de la negación, en la línea de lo propuesto por N. Kadmon y F. Landam (1993), M. Israel (1996), A. Giannakidou (1998), U. Lahiri (1998), L. Tovena (1998) y G. Chierchia (2004), (2006) para los términos de polaridad negativa, afectiva y/o modal y en R. González Rodríguez (2008a), (2008b) para los términos de polaridad positiva. Como señalamos anteriormen-

16 No recurrimos al cuantificador apenas para comprobar el comportamiento de los modificadores de aproximación y precisión con respecto a los contextos negativos porque pertenece, precisamente, a ese paradigma. 
te, los modificadores de aproximación y precisión se caracterizan por fijar el parámetro de granularidad que debe tomarse en consideración al interpretar una expresión numeral (Sauerland y Stateva, en prensa). Aquellos que restringen la denotación del numeral al que acompañan, los de precisión, obligan a interpretarlo con respecto a la granularidad más sutil; aquellos que hacen más imprecisa la denotación del numeral, los de aproximación, fuerzan la lectura en que la granularidad relevante es la más basta, como ilustramos en (10), que repetimos aquí como (35) para mayor comodidad:

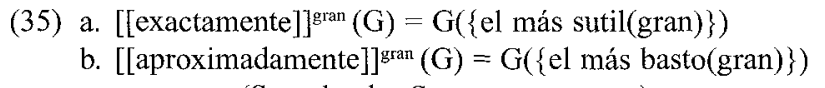

(Sauerland y Stateva, en prensa)

De este modo, lo que diferencia a las oraciones de (36) es el parámetro de granularidad con respecto al cual se interpreta la expresión numeral. La construcción de (36a) es ambigua, ya que su significado puede ser equivalente tanto al de (36b) como al de (36c), dependiendo de si el parámetro que se toma en consideración es el más sutil o el más basto, respectivamente. Las oraciones de (36b) y (36c), en cambio, se interpretan siempre de acuerdo con un mismo parámetro de granularidad. La primera de ellas con respecto al más sutil, de forma que las hormigas que había en el agujero no eran ni una más ni una menos que cien; la segunda con respecto al más basto, por lo que las hormigas que se encontraban en el agujero constituían una cantidad cercana a la denotada por ese número:

(36) a. En ese agujero había cien hormigas.

b. En ese agujero había exactamente cien hormigas.

c. En ese agujero había aproximadamente cien hormigas.

A partir de este análisis es posible tanto explicar la imposibilidad que presentan los modificadores de aproximación de ser el foco de la negación como la compatibilidad de los modificadores de precisión con la negación. Pero antes de exponer nuestra hipótesis, consideremos qué sucede cuando el foco de la negación es una expresión numeral. Obsérvese el ejemplo de (37):

(37) Juan no ha recorrido diez kilómetros.

'Existe un número de kilómetros, tal que Juan ha recorrido ese número de kilómetros, y ese número no es diez.'

a. $\operatorname{gran}_{\text {sutil }}(10)=\{10\}$

b. $\operatorname{gran}_{\text {medio }}(10)=\left\{9^{\prime} 90,9^{\prime} 91,9^{\prime} 92, \ldots . ., 10, \ldots . ., 10^{\prime} 08,10^{\prime} 09,10^{\prime} 10\right\}$

c. $\operatorname{gran}_{\text {basto }}(10)=\left\{8^{\prime} 50,8^{\prime} 51,8^{\prime} 52, \ldots \ldots ., 10, \ldots \ldots . ., 11^{\prime} 48,11^{\prime} 49,11^{\prime} 50\right\}$

La lectura resultante de (37) cuando el numeral es el foco de la negación es la que aparece parafraseada debajo del ejemplo, donde se niega que el número de kilómetros recorrido sea diez. En consecuencia, si la interpretación del numeral es, por ejemplo, la de (a), el número de kilómetros recorridos será distinto del allí expuesto, esto es, del que forma parte de la granularidad sutil. En caso de que el parámetro con respecto al cual se interprete el numeral sea el de (b) o el de (c), la situación es la misma: se niega que alguno de los valores que integran los intervalos correspondientes a esas granularidades denote el número de kilómetros recorridos por Juan. 
Veamos ahora qué sucede si introducimos un modificador de los que estamos estudiando en esa oración negativa. Comencemos, en primer lugar, con los que son incompatibles con la negación, es decir, con los modificadores de aproximación (cfr. (38)). La presencia de esos elementos desencadena la mala formación de la oración, que no puede interpretarse de acuerdo con la paráfrasis expuesta debajo del ejemplo, puesto que los modificadores de aproximación no pueden ser el foco de la negación:

(38) *Juan no ha recorrido aproximadamente diez kilómetros. \#'Existe un número de kilómetros, tal que Juan ha recorrido ese número de kilómetros, y ese número no es aproximadamente diez.'

Aproximadamente obliga a interpretar el numeral con respecto al parámetro de granularidad más basto (Sauerland y Stateva, en prensa). Al refutarse ese adverbio, se niega, por tanto, que los valores contenido en el intervalo al que refiere esa granularidad satisfagan el contenido proposicional de la oración (cfr. (39a)). De la refutación de esos valores se sigue, además, que los contenidos en las extensiones asociadas a alguno de los otros parámetros sí satisfacen el contenido proposiconal (cfr. (39b)):

(39) $[[\neg$ aproximadamente $\mathrm{x}(\mathrm{P})]]=1 \mathrm{ssi}$

$$
\begin{array}{lll}
\text { a. } & \neg \mathrm{P}\left(\operatorname{gran}_{\text {el más basto }}(\mathrm{X})\right) & \text { (aserción) } \\
\text { b. } & \mathrm{P}\left(\operatorname{gran}_{\text {sutil }}(\mathrm{X})\right) \vee \mathrm{P}\left(\text { gran }_{\text {medio }}(\mathrm{X})\right) & \text { (presuposición) }
\end{array}
$$

El hecho de que la negación de un determinado parámetro presuponga la afirmación de alguno de los otros no difiere en absoluto de lo que sucede cuando se niega otro tipo de constituyente. Así, de la interpretación de No ha llegado Juan en la que la negación incide sobre el sujeto (cfr. No es Juan el que ha llegado) se sigue que ha llegado un individuo distinto de Juan; en otras palabras, que ha llegado alguien, pero que no se trata de Juan. Del mismo modo, cuando se niega un modificador como aproximadamente se refuta el parámetro de granularidad al que se asocia y, por ende, que los valores contenidos en el intervalo que determina satisfagan el contenido proposicional de la oración. La negación de este parámetro conlleva la afirmación de alguno de los otros, es decir, el que los valores que forman parte de los intervalos vinculados con alguno de los otros parámetros satisfacen el contenido proposicional de la oración. Así, en la oración de (38), repetida aquí como (40), se niega que el número de kilómetros recorridos por el sujeto de predicación sea alguno de los que comprendidos entre $8^{\prime} 50$ y $11^{\prime} 50$, puesto que esos son los valores contenidos en la extensión de la escala asociada a la granularidad fijada por aproximadamente, la más basta (cfr. (40a)), mientras que se afirma que el número de kilómetros que ha recorrido se corresponde con alguno de los valores contenidos en las extensiones determinadas por las otras granularidades, ya sea una sutil o una media (cfr. (40b)):

(40) *Juan no ha recorrido aproximadamente diez kilómetros. \#'Existe un número de kilómetros, tal que Juan ha recorrido ese número de kilómetros, y ese número no es aproximadamente diez.'

a. $\neg \operatorname{gran}_{\mathrm{el} \text { más basto }}(10)=\left\{8^{\prime} 50,8^{\prime} 51,8^{\prime} 52, \ldots . ., 10, \ldots \ldots ., 11^{\prime} 48,11^{\prime} 49,11^{\prime} 50\right\}$ b. $\operatorname{gran}_{\text {sutil }}(10)=\{10\} \vee$ gran $_{\text {medio }}(10)=\left\{9^{\prime} 90,9^{\prime} 91,9^{\prime} 92, \ldots \ldots, 10, \ldots \ldots, 10^{\prime} 08\right.$, $\left.10 \times 09,10^{\prime} 10\right\}$ 
El problema que surge al negar un modificador de aproximación es que el parámetro de granularidad más basto contiene todos los valores que forman parte de las otras granularidades; en otras palabras, los valores de la granularidad sutil y de la media son un subconjunto de los de la granularidad más basta. Ello provoca que se nieguen y se afirmen al mismo tiempo los valores que conforman ese subconjunto, dependiendo de si evaluamos el numeral con respecto al parámetro más sutil o a los otros, respectivamente. La oración de (40) es, por tanto, agramatical porque de ella se sigue que el número de kilómetros recorridos por Juan es alguno de los comprendidos entre 9'90 y 10'10, puesto que esos son los valores contenidos en las granularidades sutil y media (cf. (40b)) y, al mismo tiempo, que no es ninguno de esos valores, ya que esos forman parte del intervalo señalado por el parámetro más basto (cf. (40a)). La imposibilidad que presentan los modificadores de aproximación de ser el foco de la negación responde a que cuando son el elemento refutado se afirma y se niega al mismo tiempo ciertos valores, lo que constituye una contradicción.

Una vez explicada la incompatibilidad de los modificadores de aproximación y la negación, pasemos a explicar por qué los modificadores de precisión no se comportan como términos de polaridad positiva. Cuando estos modificadores constituyen el foco de la negación, lo que se niega es que los valores que integran el intervalo referido por el parámetro de granularidad más sutil satisfagan el contenido proposicional de la oración y, en consecuencia, se afirma que lo hacen los valores comprendidos en la extensión correspondiente a alguno de los otros parámetros, tal como se ilustra en (41), que recoge la denotación de la negación del aproximativo:

$$
\begin{aligned}
& {[[\neg \text { exactamente } \mathrm{x}(\mathrm{P})]]=1 \mathrm{ssi}} \\
& \text { a. } \neg \mathrm{P}\left(\operatorname{gran}_{\text {el mas sutil }}(\mathrm{x})\right) \quad(\text { aserción }) \\
& \text { b. } \quad \mathrm{P}\left(\operatorname{gran}_{\text {medio }}(\mathrm{X})\right) \vee \mathrm{P}\left(\operatorname{gran}_{\text {basto }}(\mathrm{X})\right) \quad \text { (presuposición) }
\end{aligned}
$$

De acuerdo con esta propuesta, en la oración de (42) se niega que Juan haya recorrido los kilómetros expresados por los valores contenidos en la extensión determinada por la granularidad más sutil (cfr. (42a)), afirmándose, en consecuencia, que el número de kilómetros recorridos es alguno de los que forman parte de la extensión de la granularidad media o basta (cfr. $(42 b))$ :

(42) Juan no ha recorrido exactamente diez kilómetros.

'Existe un número de kilómetros, tal que Juan ha recorrido ese número de kilómetros, y ese número no es exactamente diez.'
a. $\neg$ gran $_{\text {el más sutil }}=\{10\}$
b. $\operatorname{gran}_{\text {medio }}(10)=\left\{9^{\prime} 90,9^{\prime} 91,9^{\prime} 92, \ldots . ., 10, \ldots . ., 10^{\prime} 08,10^{\prime} 09,10^{\prime} 10\right\} \vee$ $\operatorname{gran}_{\text {basto }}(10)=\left\{8^{\prime} 50,8^{\prime} 51,8^{\prime} 52, \ldots . ., 10, \ldots . . ., 11^{\prime} 48,11^{\prime} 49,11^{\prime} 50\right\}$

La negación de exactamente no desencadena la agramaticalidad de la construcción porque, a diferencia de lo que sucede con aproximadamente, el parámetro de granularidad fijado por ese modificador, el más sutil, no está integrado por un conjunto de valores tal que un subconjunto de ellos se corresponda con los integrados en las otras granularidades; en otras palabras, el parámetro de granularidad negado no contiene todos los valores que conforman los parámetros que se afirman. A pesar de que el valor negado, diez, forma parte de los parámetros que se afirman, estos contienen otros valores, los cuales pueden ser afir- 
mados sin que se produzca contradicción alguna. En (42) se niega, por tanto, que el número de kilómetros recorridos por Juan sea diez y se afirma que lo es alguno de los contenidos en el parámetro medio o en el basto. Aunque el valor seleccionado de esos parámetros no puede ser diez, puede serlo cualquiera de los otros que los integran sin dar lugar a ninguna contradicción.

Nuestra propuesta explica, además, que los modificadores de aproximación puedan concurrir con la negación cuando no son su foco, como sucede, por ejemplo, en (43), donde la cláusula final (cfr. (43a)) y la causal (cfr. (43b)) en subjuntivo son los elementos refutados:

(43) a. Juan no ha recorrido aproximadamente diez kilómetros para que su entrenador se quede impresionado.

b. Juan no ha recorrido aproximadamente diez kilómetros porque esté preparándose la oposición.

En las oraciones de (43) se afirma, por una parte, que Juan ha recorrido aproximadamente diez kilómetros y se niega, por otra, que lo haya hecho para que su entrenador se quede impresionado (cfr. (43a)) o porque esté preparándose la oposición (cfr. (43b)). La contribución semántica realizada por el adverbio no se ve, por tanto, afectada por la negación. Dado que el modificador no es el foco de la negación, ninguna de esas construcciones presenta la incongruencia que surge cuando se niega que los valores contenidos en el intervalo asociado al parámetro de granularidad más basto satisfacen el contenido proposicional de la oración. De ello se sigue que los modificadores de aproximación no sean rechazados en los contextos negativos cuando no constituyen el elemento refutado.

En resumen, la negación de los modificadores de aproximación conlleva la del parámetro de granularidad con que se vinculan y la afirmación de alguno de los otros. En el caso de los modificadores de aproximación, eso da lugar a una contradicción. La razón es que todos los valores disponibles en los parámetros de granularidad afirmados forman también parte de la granularidad negada y, en consecuencia, son negados y afirmados al mismo tiempo. Cuando se niega un modificador de precisión no surge ese problema, ya que tan solo uno de los valores que integran las granularidades afirmadas forma parte también de la granularidad negada. Al existir otros valores disponibles en las granularidades afirmadas, puede refutarse el valor de la granularidad negada y afirmarse que alguno de los correspondientes a las otras satisface el contenido proposicional de la correspondiente oración afirmativa sin que se esté negando y afirmando un valor al mismo tiempo.

\section{Conclusiones}

En este trabajo hemos estudiado el comportamiento que presentan los modificadores de aproximación y precisión en las oraciones negativas: los primeros, a diferencia de los segundos, son incompatibles con la negación. En primer lugar, hemos determinado que los modificadores de aproximación son rechazados únicamente si son el foco de la negación, de forma que pueden aparecer en una oración negativa siempre y cuando no sean el elemento refutado. Además, hemos ofrecido datos que indican que esos modificadores tampoco son incompatibles con otros inductores de polaridad negativa distintos de la negación, como, por ejemplo, el cuantificador pocos o las construcciones comparativas. En segundo lugar, 
hemos propuesto que la distribución que presentan los modificadores de aproximación y precisión se deriva de sus propiedades semánticas. Basándonos en el análisis semántico desarrollado por U. Sauerland y P. Stateva (en prensa) para los modificadores de aproximación y precisión, hemos defendido que los primeros no pueden ser el foco de la negación porque de su refutación se sigue que ciertos valores que forman parte del intervalo de la escala al que señala el numeral satisfacen y no satisfacen el contenido proposicional de la oración, lo que constituye una contradicción; la negación de los segundos, en cambio, no plantea ese problema.

\section{Referencias bibliográficas}

Borgonovo, C. (2002): "Mood and Focus", Cuadernos de Lingüística, 9, Madrid, Instituto Universitario Ortega y Gasset, 43-63.

Bosque, I. (1980a): "La preposición sin", Lingüistica Hispánica, 3, 71-85.

Bosque, I. (1980b): Sobre la negación. Madrid, Cátedra.

Bosque, I. (1989): Las categorias gramaticales. Madrid, Síntesis.

Bosque, I. (1990): "Las bases gramaticales de la alternancia modal. Repaso y balance", en Bosque. I. (ed.): Indicativo y subjuntivo. Madrid, Taurus, 13-66.

Chierchia, G. (2004): "Scalar Implicatures, Polarity Phenomena, and the Syntax/Pragmatics Interface", en Belleti, A. (ed.): Structures and Beyond. The Cartography of Syntactic Structures. Oxford, Oxford University Press, 39-103.

Chierchia, G. (2006): "Broaden Your Views: Implicatures of Domain Widening and the "Logicality" of Language", Linguistic Inquiry, 37, 535-590.

García-Medall, J. (1993): "Sobre casi y otros aproximativos", Dicenda. Cuadernos de Filologia Hispánica, 11, 153-170.

García-Page, M. (1995): "El adverbio de exactitud y aproximación", Anuario de lingütística hispánica, XI, 169-180.

Giannakidou, A. (1998): Polarity Sensitivity as (Non)veridical Dependency. Amsterdam, John Benjamins.

González Rodríguez, R. (2008a): La polaridad positiva en español, tesis doctoral, Instituto Universitario de Investigación Ortega y Gasset/ Universidad Complutense de Madrid.

González Rodríguez, R. (2008b): "Wh-Phrases as Positive Polarity Items", Catalan Journal of Lin guistics, 7, 91-116.

Horn, L. R. (1985): "Metalinguistic Negation and Pragmatic Ambiguity", Language, 61:1, 121-174.

Israel, M. (1996): "Polarity sensitivity as lexical semantics", Linguistics and Philosophy, 19, 619-666.

Kadmon, N. y F. Landman (1993): "Any", Linguistics and Philosophy, 1, 3-44.

Kennedy, C. (2007): "Vagueness and grammar: the semantics of relative and absolute gradable adjectives", Linguistics and Philosophy, 30:1, 1-45.

Krifka, M. (2007): "Approximative Interpretations of Number Words: A Case of Strategic Communication", en Bouma, G et alii (eds.): Cognitive foundations of interpretation. Amsterdam, Koninklijke, Nederlandse Akademie van Wetenschapen, 111-126.

Lahiri, U. (1998): "Focus and Negative Polarity in Hindi", Natural Language Semantics, 6, 57-125.

Laka, I. (1990): Negation in Syntax: On the Nature of Functional Categories and Projections, tesis doctoral, MIT.

Lakoff, G. (1972): "Hedges: a study in meaning criteria and the logic of fuzzy concepts", Chicago Linguistic Society, 8, 183-228.

Lasersohn, P. (1999): "Pragmatic Halos", Language, 75, 522-551.

Moreno Cabrera, J. C. (1984): "Observaciones sobre la sintaxis de «casi »", Dicenda. Cuadernos de

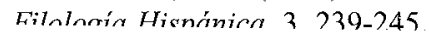


Pinkal, M. (1995): Logic and Lexicon. Dordrecht, Kluwer.

Quer, J. (1998): Mood at the Interface. La Haya, Holland Academic Graphics.

Sauerland, U. y P. Stateva (en prensa): "Scalar vs. Epistemic Vagueness: Evidence from Approximators", en Gibson, M. y T. Friedman (eds.): Proceedings of Semantics and Linguistic Theory, 17. Ithaca, N.Y., Cornell University.

Tovena, L. (1998): The fine structure of polarity items. Nueva York, Garland. Williamson, T. (1994): Vagueness. London, Routledge. 